\title{
Accurate Asymptotic Formulas for Eigenvalues of a Boundary-value Problem of Fourth Order
}

\author{
Karwan H. F. Jwamer ${ }^{1, *}$, Hawsar Ali HR ${ }^{2}$ \\ ${ }^{1}$ Department of Mathematics, School of Science, University of Sulaimani, Iraq \\ ${ }^{2}$ Department of Mathematics, School of Science Education, University of Sulaimani, Iraq
}

Copyright (C) 2015 by authors, all rights reserved. Authors agree that this article remains permanently open access under the terms of the Creative Commons Attribution License 4.0 International License

\begin{abstract}
This paper deals with the behavior of the solution and asymptotic behaviors of eigenvalues of a fourth order boundary value problem, having the following definition: $y^{(4)}+p_{3}(x) y^{\prime}+p_{4}(x) y=\lambda^{4} \rho(x) y$ (1) with boundary conditions: $y^{(j)}(0)-y^{(j)}(\pi)=0, j=$ $0,1,2,3$ Where $p_{3}(x)$ and $p_{4}(x)$ are real valued functions and $\rho(x)=1$, and $\lambda$ is a spectral parameter in which $\lambda=\sigma+i \tau, \sigma, \tau \in R, i=\sqrt{-1}, \tau \neq 0$. Here it has been assumed that $p_{4}(x) \in C[0, a]$ and $p_{3}(x) \in C^{2}[0, a]$.
\end{abstract}

Keywords Spectral Problem, Eigenvalues , Eigen Functions, Asymptotic Formulas

\section{Introduction}

In [1] Sturm and Liouville first pursued this issue in 19th century. They examined the issue in a finite interval and for second order operators. Then, in 1908, after the satisfaction of the regular boundary conditions, Birkhoff studied the distribution of differential operators in a finite interval but for arbitrary orders. In [6] Jwamer using this concept defined fourth order of differential operator. In [7] Menken Hamza using the asymptotic formulas in the case $y^{(4)}+q(x) y=$ $\lambda^{4} y, 0<x<\pi$ was proved.

\section{Study the Behavior of the Solutions}

In this section, we try to estimate the behavior of the solution to the fourth order boundary value problem described above, and to find their conditions $A_{i}(x), i=$ $0,1,2,3,4$ later.

\section{Theorem 2.1}

Assume $\lambda \in T_{k}$ or $\lambda \in \overline{T_{k}}, k=0,1,2,3$ and $w_{k}, k=$ $0,1,2,3$. Then there exist linear independent solutions $y_{k}(x, \lambda), k=0,1,2,3,4$ of equation (1) with sufficiently large $|\lambda|$, in such a way when $j=0,1,2,3$ uniformly in $0 \leq x \leq \pi$ satisfying the relation

$$
y_{k}^{(j)}(x, \lambda)=\left(i \lambda w_{k}\right)^{j} e^{i \lambda w_{k} x}\left[\sum_{i=0}^{4} \frac{A_{i}^{(j)}(x)}{\lambda^{i}}+O\left(\frac{1}{\lambda^{5}}\right)\right]
$$

Such that

$$
\begin{gathered}
A_{0}(x)=1, A_{1}(x)=0, A_{2}(x)=\frac{-1}{4\left(i w_{k}\right)^{2}} \int_{0}^{x} p_{3}(t) d t, \\
A_{3}(x)=\frac{1}{4\left(i w_{k}\right)^{3}} \int_{0}^{x}\left(\frac{3}{2} p_{3}^{\prime}(t)-p_{4}(t)\right) d t \text { and } \\
A_{4}(x)=\frac{-1}{16} \int_{0}^{x}\left(5 p_{3}^{\prime \prime}(t)-6 p_{4}^{\prime}(t)+\frac{4 p_{3}(t)}{\left(i w_{k}\right)^{2}} A_{2}(t)\right) d t
\end{gathered}
$$

\section{Proof}

From [8], it was proved that the solutions of equation (1) for sufficient large $|\lambda|$ which can be written in the form

$$
\begin{gathered}
y_{k}(x, \lambda)=e^{\lambda \int_{0}^{x} \varphi_{k}(t) d t}\left(\sum_{i=0}^{4} \frac{A_{i}(x)}{\lambda^{i}}+O\left(\frac{1}{\lambda^{5}}\right)\right) \\
\text { But } e^{\lambda \int_{0}^{x} \varphi_{k}(t) d t}=e^{\lambda \int_{0}^{x} i w_{k} \sqrt[4]{\rho(t)} d t} \\
=e^{i \lambda w_{k} \int_{0}^{x} d t}=e^{i \lambda w_{k} x} \\
y_{k}(x, \lambda)=e^{i \lambda w_{k} x}\left(\sum_{i=0}^{4} \frac{A_{i}(x)}{\lambda^{i}}+O\left(\frac{1}{\lambda^{5}}\right)\right)
\end{gathered}
$$

By differentiating (2) up to fourth order with respect to $x$, the following relations are obtained:

$$
\begin{gathered}
y_{k}^{\prime}(x, \lambda)=\left(i \lambda w_{k}\right) e^{i \lambda w_{k} x}\left[A_{0}(x)+\sum_{i=0}^{3} \frac{1}{\lambda^{i+1}}\left(A_{i+1}(x)+\right.\right. \\
\left.\left.\frac{A_{i}^{\prime}(x)}{i w_{k}}\right)+O\left(\frac{1}{\lambda^{5}}\right)\right]
\end{gathered}
$$

$$
\begin{gathered}
y_{k}^{\prime \prime}(x, \lambda)=\left(i \lambda w_{k}\right)^{2} e^{i \lambda w_{k} x}\left[A_{0}(x)+\frac{1}{\lambda}\left(A_{1}(x)+2 \frac{A_{0}^{\prime}(x)}{i w_{k}}\right)\right. \\
+\sum_{i=0}^{2} \frac{1}{\lambda^{i+2}}\left(A_{i+2}(x)+2 \frac{A_{i+1}^{\prime}(x)}{i w_{k}}\right. \\
\left.\left.+\frac{A_{i}^{\prime \prime}(x)}{\left(i w_{k}\right)^{2}}\right)+O\left(\frac{1}{\lambda^{5}}\right)\right]
\end{gathered}
$$




$$
\begin{array}{r}
y_{k}^{\prime \prime \prime}(x, \lambda)=\left(i \lambda w_{k}\right)^{3} e^{i \lambda w_{k} x}\left[A_{0}(x)+\frac{1}{\lambda}\left(A_{1}(x)+3 \frac{A_{0}^{\prime}(x)}{i w_{k}}\right)\right. \\
+\frac{1}{\lambda^{2}}\left(A_{2}(x)+3 \frac{A_{1}^{\prime}(x)}{i w_{k}}+3 \frac{A_{0}^{\prime \prime}(x)}{\left(i w_{k}\right)^{2}}\right) \\
+\sum_{i=0}^{1} \frac{1}{\lambda^{i+3}}\left(A_{i+3}(x)+2 \frac{A_{i+2}^{\prime}(x)}{i w_{k}}\right. \\
\left.\left.+\frac{A_{i+1}^{\prime \prime}(x)}{\left(i w_{k}\right)^{2}}\right)+O\left(\frac{1}{\lambda^{5}}\right)\right] \\
\left.4 \frac{A_{0}^{\prime}(x)}{i w_{k}}\right)+\frac{1}{\lambda^{2}}\left(A_{2}(x)+4 \frac{A_{1}^{\prime}(x)}{i w_{k}}+6 \frac{A_{0}^{\prime \prime}(x)}{\left(i w_{k}\right)^{2}}\right)+\frac{1}{\lambda^{3}}\left(A_{3}(x)+\right. \\
\left.4 \frac{A_{2}^{\prime}(x)}{i w_{k}}+6 \frac{A_{1}^{\prime \prime}(x)}{\left(i w_{k}\right)^{2}}+4 \frac{A_{0}^{\prime \prime \prime}(x)}{\left(i w_{k}\right)^{3}}\right)+\frac{1}{\lambda^{4}}\left(A_{4}(x)+4 \frac{A_{3}^{\prime}(x)}{i w_{k}}+\right. \\
\left.\left.6 \frac{A_{2}^{\prime \prime}(x)}{\left(i w_{k}\right)^{2}}+4 \frac{A_{1}^{\prime \prime \prime}(x)}{\left(i w_{k}\right)^{3}}+\frac{A_{0}^{(4)}(x)}{\left(i w_{k}\right)^{4}}\right)+O\left(\frac{1}{\lambda^{5}}\right)\right]
\end{array}
$$

Now, the resultant equations (2),(3) and (4) are substituted by the equation (1) to obtain the results.

\section{Asymptotic Behaviors of Eigenvalues to the Problem}

The primary purpose of this section is to study the asymptotic behaviors of eigenvalues with different cases of the following types:

$$
\begin{gathered}
\text { (1) If } \int_{0}^{\pi} p_{i}(t) d t=0 \text { for } i=3,4 \\
\text { then } A_{2}(\pi)=0, \\
A_{3}(\pi)=\frac{3}{8\left(i w_{k}\right)^{3}}\left[p_{3}(\pi)-p_{3}(0)\right] \\
A_{4}(\pi)=\frac{-1}{16}\left[5\left(p_{3}^{\prime}(\pi)-p_{3}^{\prime}(0)\right)-6\left(p_{4}(\pi)-p_{4}(0)\right)\right]
\end{gathered}
$$

(2) If a zero is a root of $p_{3}(x)$,

$$
\text { then } p_{3}(0)=0 \text {. }
$$

to the given spectral problem by the theorem:

\section{Theorem 3.1}

Asymptotic behavior of eigenvalues for sufficiently large $\lambda$ of the spectral boundary value problem in the sector $T_{k}$ has the form:

$$
\begin{aligned}
& \lambda_{m}=\frac{2 k}{(i)^{k+4}}, k=0,1,2, \ldots \\
& \lambda_{m}=\sqrt[4]{\frac{\frac{3}{2} p_{4}(0)-\frac{5}{4} p_{3}^{\prime}(0)}{1+(2 k)^{5}}}
\end{aligned}
$$

Proof

$$
\begin{gathered}
y_{k}(0, \lambda)=\left[1+O\left(\frac{1}{\lambda^{5}}\right)\right] \\
y_{k}^{\prime}(0, \lambda)=\left(i \lambda w_{k}\right)\left[1+\frac{1}{\lambda^{4}}\left(\left[\frac{3}{8} p_{3}^{\prime}(0)-\frac{p_{4}(0)}{4}\right]\right)+O\left(\frac{1}{\lambda^{5}}\right)\right]
\end{gathered}
$$

$$
\begin{array}{r}
y_{k}^{\prime \prime}(0, \lambda)=\left(i \lambda w_{k}\right)^{2}\left[1+\frac{\left(\frac{1}{2} p_{3}^{\prime}(0)-\frac{1}{2} p_{4}(0)\right)}{\lambda^{4}}+O\left(\frac{1}{\lambda^{5}}\right)\right] \\
y_{k}^{\prime \prime \prime}(0, \lambda)=\left(i \lambda w_{k}\right)^{3}\left[1+\frac{\left(\frac{3}{8} p_{3}^{\prime}(0)-\frac{3}{4} p_{4}(0)\right)}{\lambda^{4}}+O\left(\frac{1}{\lambda^{5}}\right)\right] \\
y_{k}(\pi, \lambda)=e^{i \lambda w_{k} \pi\left[1+\frac{1}{\lambda^{3}}\left(\frac{3 p_{3}(\pi)}{8\left(i w_{k}\right)^{3}}\right)\right.} \\
+\frac{1}{\lambda^{4}}\left(\frac { - 1 } { 1 6 } \left[5\left(p_{3}^{\prime}(\pi)-p_{3}^{\prime}(0)\right)-6 p_{4}(\pi)\right.\right. \\
\left.\left.\left.\left.+6 p_{4}(0)\right)\right]\right)+O\left(\frac{1}{\lambda^{5}}\right)\right]
\end{array}
$$

$$
\begin{aligned}
y_{k}^{\prime}(\pi, \lambda)= & \left(i \lambda w_{k}\right) e^{i \lambda w_{k} \pi}\left[1+\frac{1}{\lambda^{3}}\left(\frac{p_{3}(\pi)}{8\left(i w_{k}\right)^{3}}\right)\right. \\
& +\frac{1}{\lambda^{4}}\left(\frac { 1 } { 1 6 } \left[p_{3}^{\prime}(\pi)+5 p_{3}^{\prime}(0)\right.\right. \\
& \left.\left.\left.+2 p_{4}(\pi)-6 p_{4}(0)\right]\right)+O\left(\frac{1}{\lambda^{5}}\right)\right]
\end{aligned}
$$

$$
\begin{aligned}
y_{k}^{\prime \prime}(\pi, \lambda)= & \left(i \lambda w_{k}\right)^{2} e^{i \lambda w_{k} \pi}\left[1+\frac{1}{\lambda^{3}}\left(\frac{-p_{3}(\pi)}{8\left(i w_{k}\right)^{3}}\right)\right. \\
& +\frac{1}{\lambda^{4}}\left(\frac { 1 } { 1 6 } \left[3 p_{3}^{\prime}(\pi)+5 p_{3}^{\prime}(0)\right.\right. \\
& \left.\left.\left.-2 p_{4}(\pi)-6 p_{4}(0)\right]\right)+O\left(\frac{1}{\lambda^{5}}\right)\right]
\end{aligned}
$$

$$
\begin{aligned}
y_{k}^{\prime \prime \prime}(\pi, \lambda)= & \left(i \lambda w_{k}\right)^{3} e^{i \lambda w_{k} \pi}\left[1+\frac{1}{\lambda^{3}}\left(\frac{-3 p_{3}(\pi)}{8\left(i w_{k}\right)^{3}}\right)\right. \\
& +\frac{1}{\lambda^{4}}\left(\frac { 1 } { 1 6 } \left[p_{3}^{\prime}(\pi)+5 p_{3}^{\prime}(0)\right.\right. \\
& \left.\left.\left.-6\left(p_{4}(\pi)+p_{4}(0)\right)\right]\right)+O\left(\frac{1}{\lambda^{5}}\right)\right]
\end{aligned}
$$

$$
\Delta(\lambda)=\left|\begin{array}{llll}
U_{0}\left(y_{0}\right) & U_{0}\left(y_{1}\right) & U_{0}\left(y_{2}\right) & U_{0}\left(y_{3}\right) \\
U_{1}\left(y_{0}\right) & U_{1}\left(y_{1}\right) & U_{1}\left(y_{2}\right) & U_{1}\left(y_{3}\right) \\
U_{2}\left(y_{0}\right) & U_{2}\left(y_{1}\right) & U_{2}\left(y_{2}\right) & U_{2}\left(y_{3}\right) \\
U_{3}\left(y_{0}\right) & U_{3}\left(y_{1}\right) & U_{3}\left(y_{2}\right) & U_{3}\left(y_{3}\right)
\end{array}\right|
$$

Where $U_{j}\left(y_{k}\right)=y_{k}^{(j)}(\pi)-y_{k}^{(j)}(0), \quad(j, k=0,1,2,3)$.

$$
\begin{gathered}
U_{0}\left(y_{k}\right)=y_{k}(\pi)-y_{k}(0) \\
=e^{i \lambda w_{k} \pi}\left[1+\frac{1}{\lambda^{3}}\left(\frac{3 p_{3}(\pi)}{8\left(i w_{k}\right)^{3}}\right)\right. \\
+\frac{1}{\lambda^{4}}\left(\frac{-\left[5\left(p_{3}^{\prime}(\pi)-p_{3}^{\prime}(0)\right)-6\left(p_{4}(\pi)-p_{4}(0)\right]\right.}{16}\right) \\
\left.+O\left(\frac{1}{\lambda^{5}}\right)\right]-\left[1+O\left(\frac{1}{\lambda^{5}}\right)\right] \quad k=0,1,2,3 \\
U_{1}\left(y_{k}\right)=y_{k}^{\prime}(\pi)-y_{k}^{\prime}(0)
\end{gathered}
$$




$$
\begin{aligned}
& U_{1}\left(y_{k}\right)=\left(i \lambda w_{k}\right) e^{i \lambda w_{k} \pi}\left[1+\frac{1}{\lambda^{3}}\left(\frac{p_{3}(\pi)}{8\left(i w_{k}\right)^{3}}\right)\right. \\
& +\frac{1}{\lambda^{4}}\left(\frac { 1 } { 1 6 } \left[p_{3}^{\prime}(\pi)+5 p_{3}^{\prime}(0)\right.\right. \\
& \left.\left.\left.+2 p_{4}(\pi)-6 p_{4}(0)\right]\right)+O\left(\frac{1}{\lambda^{5}}\right)\right] \\
& -\left(i \lambda w_{k}\right)\left[1+\frac{1}{\lambda^{4}}\left(\left[\frac{3}{8} p_{3}^{\prime}(0)-\frac{p_{4}(0)}{4}\right]\right)\right. \\
& \left.+O\left(\frac{1}{\lambda^{5}}\right)\right] k=0,1,2,3 \\
& U_{2}\left(y_{k}\right)=y_{k}^{\prime \prime}(\pi)-y_{k}^{\prime \prime}(0) \\
& U_{2}\left(y_{k}\right)=\left(i \lambda w_{k}\right)^{2} e^{i \lambda w_{k} \pi}\left[1+\frac{1}{\lambda^{3}}\left(\frac{-p_{3}(\pi)}{8\left(i w_{k}\right)^{3}}\right)\right. \\
& +\frac{1}{\lambda^{4}}\left(\frac { 1 } { 1 6 } \left[3 p_{3}^{\prime}(\pi)+5 p_{3}^{\prime}(0)\right.\right. \\
& \left.\left.\left.-2 p_{4}(\pi)-6 p_{4}(0)\right]\right)+O\left(\frac{1}{\lambda^{5}}\right)\right] \\
& -\left(i \lambda w_{k}\right)^{2}\left[1+\frac{1}{\lambda^{4}}\left(\left[\frac{1}{2} p_{3}^{\prime}(0)-\frac{1}{2} p_{4}(0)\right]\right)\right. \\
& \left.+O\left(\frac{1}{\lambda^{5}}\right)\right] k=0,1,2,3 \\
& U_{3}\left(y_{k}\right)=y_{k}^{\prime \prime \prime}(\pi)-y_{k}^{\prime \prime \prime}(0) \\
& U_{3}\left(y_{k}\right)=\left(i \lambda w_{k}\right)^{3} e^{i \lambda w_{k} \pi}\left[1+\frac{1}{\lambda^{3}}\left(\frac{-3 p_{3}(\pi)}{8\left(i w_{k}\right)^{3}}\right)\right. \\
& +\frac{1}{\lambda^{4}}\left(\frac { 1 } { 1 6 } \left[p_{3}^{\prime}(\pi)+5 p_{3}^{\prime}(0)\right.\right. \\
& \left.\left.\left.-6\left(p_{4}(\pi)+p_{4}(0)\right)\right]\right)+O\left(\frac{1}{\lambda^{5}}\right)\right] \\
& -\left(i \lambda w_{k}\right)^{3}\left[1+\frac{1}{\lambda^{4}}\left(\left[\frac{3}{8} p_{3}^{\prime}(0)-\frac{3}{4} p_{4}(0)\right]\right)\right. \\
& \left.+O\left(\frac{1}{\lambda^{5}}\right)\right] k=0,1,2,3 \\
& \Delta(\lambda)=\left(i \lambda w_{k}\right)^{6} e^{4 i \lambda w_{k} \pi}\left[1+\frac{1}{\lambda^{4}}\left(\frac{1}{4}\left[5 p_{3}^{\prime}(0)-6 p_{4}(0)\right]\right)\right. \\
& \left.+O\left(\frac{1}{\lambda^{5}}\right)\right] \\
& -\left(i \lambda w_{k}\right)^{6} e^{3 i \lambda w_{k} \pi}\left[4+\frac{1}{\lambda^{4}}\left(\left[5 p_{3}^{\prime}(0)-6 p_{4}(0)\right]\right)+O\left(\frac{1}{\lambda^{5}}\right)\right] \\
& +\left(i \lambda w_{k}\right)^{6} e^{2 i \lambda w_{k} \pi}\left[6+\frac{1}{\lambda^{4}}\left(\frac{1}{2}\left[15 p_{3}^{\prime}(0)-18 p_{4}(0)\right]\right)\right. \\
& \left.+O\left(\frac{1}{\lambda^{5}}\right)\right] \\
& -\left(i \lambda w_{k}\right)^{6} e^{i \lambda w_{k} \pi}\left[4+\frac{1}{\lambda^{4}}\left(\left[5 p_{3}^{\prime}(0)-6 p_{4}(0)\right]\right)+O\left(\frac{1}{\lambda^{5}}\right)\right] \\
& +\left(i \lambda w_{k}\right)^{6}\left[1+\frac{1}{\lambda^{4}}\left(\frac{1}{4}\left[5 p_{3}^{\prime}(0)-6 p_{4}(0)\right]\right)+O\left(\frac{1}{\lambda^{5}}\right)\right]
\end{aligned}
$$

$$
\begin{aligned}
\Delta(\lambda)=\left(i \lambda w_{k}\right)^{6} & {\left[\left(e^{4 i \lambda w_{k} \pi}-4 e^{3 i \lambda w_{k} \pi}+6 e^{2 i \lambda w_{k} \pi}\right.\right.} \\
& \left.-4 e^{i \lambda w_{k} \pi}+1\right) \\
& *\left(1+\frac{1}{\lambda^{4}}\left(\frac{1}{4}\left[5 p_{3}^{\prime}(0)-6 p_{4}(0)\right]\right)\right. \\
& \left.\left.+O\left(\frac{1}{\lambda^{5}}\right)\right)\right] \\
\Delta(\lambda) & =\left(i \lambda w_{k}\right)^{6}\left(e^{i \lambda w_{k} \pi}-1\right)^{4}(1 \\
& \left.+\frac{1}{\lambda^{4}}\left(\frac{1}{4}\left[5 p_{3}^{\prime}(0)-6 p_{4}(0)\right]\right)+O\left(\frac{1}{\lambda^{5}}\right)\right)
\end{aligned}
$$

Hence, by $\Delta(\lambda)=0$, for sufficiently large $|\lambda|$, the following equations hold:

$$
e^{i \lambda w_{k} \pi}-1=0 \Rightarrow e^{i \lambda w_{k} \pi}=1 \Rightarrow \lambda_{m}=\frac{2 k}{w_{k}}
$$

but $w_{k}=(i)^{k+4}$

Hence, $\lambda_{m}=\frac{2 k}{(i)^{k+4}}, k=0,1,2, \ldots$

Or

$$
\begin{gathered}
1+\frac{1}{\lambda^{4}}\left(\frac{1}{4}\left[5 p_{3}^{\prime}(0)-6 p_{4}(0)\right]\right)+O\left(\frac{1}{\lambda^{5}}\right)=0 \\
1-\frac{1}{\lambda^{4}}\left(\frac{3}{2} p_{4}(0)-\frac{5}{4} p_{3}^{\prime}(0)\right)+O\left(\frac{1}{\lambda^{5}}\right)=0 \\
1+O\left(\frac{1}{\lambda^{5}}\right)=\frac{1}{\lambda^{4}}\left(\frac{3}{2} p_{4}(0)-\frac{5}{4} p_{3}^{\prime}(0)\right)
\end{gathered}
$$

Thus,

$$
\begin{gathered}
\lambda_{m}=\sqrt[4]{\frac{\frac{3}{2} p_{4}(0)-\frac{5}{4} p_{3}^{\prime}(0)}{1+o\left(\frac{1}{\lambda^{5}}\right)}} \\
\lambda_{m}=\sqrt[4]{\frac{\frac{3}{2} p_{4}(0)-\frac{5}{4} p_{3}^{\prime}(0)}{1+(2 k)^{5}}}
\end{gathered}
$$

\section{Conclusions}

It is found the asymptotic formula for eigenvalues to the spectral problem as defined in equation (1), the coefficients in the differential equation are real valued functions and $\rho(x)=1$ and it is assumed that $p_{4}(x) \in C[0, a]$ and $p_{3}(x) \in C^{2}[0, a]$.

\section{REFERENCES}

[1] Birkhoff, GD: boundary value and expansion problems of ordinary linear differential equations. Trans. Am. Math. Soc. 9(4), 373-395 (1908). 
[2] Fedoryuk, MV: Asymptotic Methods in Analysis. Encyclopaedia of Mathematical Science, vol. 13,pp.83-191(1989).

[3] Jwamer, K. H., Analysis of Spectral Characteristics of One Non-selfadjoint Problem with Non-Smooth Coefficients, PHD thesis, Dagestan State University, South of Russian,(2010).

[4] Karwan H.F. Jwamer and Aryan A.M, Study the Behavior of the Solution and Asymptotic Behaviors of Eigenvalues of a Six Order Boundary Value Problem, International Journal of Research and Reviews in Applied Sciences, Vol.13, Issue 3, December 2012,p.790-799, Pakistan.

[5] Karwan H.F. Jwamer and Aryan A.M, Boundedness of Normalized Eigenfunctions of the Spectral Problem in the Case of Weight Function Satisfying the Lipschitz Condition, Journal of Zankoy Sulaimani - Part A (JZS-A), Vol. 15, No.1, 2013, p.79-94.

[6] Karwan .H.F.Jwamer ,Aigounv G.A and Gajiva T.Yu, The Study of the Asymptotic Behavior of the Eigenvalues and the
Estimate for the Kernel of the Resolvent of an Irregular Boundary Value Problem Generated by a Differential Equation of Order Four on the interval [0, a], Bulletin of Dagestan State University, Natural Sciences, Makhachkala(South of Russian), Vol.4,No.4 ,2007,p. 93-97.

[7] Menken Hamza, Accurate Asymptotic Formulas for Eigenvalues and Eigenfunctions of a Boundary-Value Problem of Fourth Order, Boundary Value Problems 2010,720235 (28 November 2010), Springer, pp.1-21.

[8] Naimark .M.A, Linear Differential Operators, New York, USA, 1968 .

[9] Tamarkin.Ya.D, About Some General Problems of Theory of Ordinary Linear Differential Equations and About Decomposition of Arbitrary Functions in Series, Petrograd,1917.

[10] Tamila. Yu., G., Analysis of Spectral Characteristics of One Non-selfadjoint Problem with Smooth Coefficients, PhD thesis, Dagestan State University, South of Russian, (2010). 